\title{
The research on the UAV positioning method using the POS and improved image matching
}

\author{
Zhang Yan ${ }^{1}$, Jiang Panpan ${ }^{2}$,Li Jianzeng ${ }^{1}$, Li Deliang ${ }^{1}$, Li Pengjun ${ }^{1}$, DuYulong ${ }^{1}$ \\ ${ }^{1}$ Ordnance Engineering College, Shijiazhuang 050003, China \\ ${ }^{2}$ College of Geo-exploration Science and Technology, Jilin University, Changchun 130026, China
}

Keywords: Image Registration; UAV; Target location; POS; Optical flow

\begin{abstract}
Aiming at the problem of low accuracy of the target for one UAV(Unmanned Aerial Vehicle), according to the UAV's characteristics of UAV aerial images, POS dates and satellite digital map, a UAV image matching and targeting method is presented based on POS. At first, using the Gaussian pyramid optical flow method, the UAV aerials or video frames are real-time stitched. Then, taking advantage of POS date, the approximate area of the entire aerials or video frames on the satellite digital map are determined based on the formulated search strategy. Finally, making use of the improved SIFT algorithm, the aerial photos(video frames) and satellite digital map in this range are matched to complete the targeting. The results proved that this method can achieve a wide range of real-time two-dimensional positioning without ground control points, while the precision and speed has large improvement, which is an effective method for target location utilizing image matching.
\end{abstract}

\section{Introduction ${ }^{1}$}

Currently, how to achieve positioning quickly and exactly for the goal is one of the core issues in the field of UAV research. The positioning accuracy of the traditional UAV map-matching and the atmospheric height measurements parameters method is under the influence of the underlay, low precision, and the application range is limited ${ }^{[1]}$. In recent years, with the rapid development of the related computer vision algorithm performance ${ }^{[2]}$ and UAV's POS technology, which makes it possible to use the image matching to achieve precise positioning, and the matching algorithm is the core content.

Because Gaussian pyramid optical flow algorithm exists problems, many improved algorithms from different angles are proposed by scholars at home and abroad, mainly focusing in the generation process of simplification feature vector, which solve the matching problem of noise images and affine images. However, in the algorithm, the threshold value of the distance ratio can't be adaptive, the matching accuracy is not guaranteed.

In this paper, the feature that the UAV sensors can provide aerial imagery and POS information at the same time is used, as well as the advantages of a satellite digital map widely applied. Mainly for the two-dimensional positioning target, based on image matching, the UAV targeting method is proposed. without the condition of leading to the ground control points, using POS auxiliary aerial triangulation measurement and Gaussian pyramid optical flow matching method, the searching strategy is developed to complete adaptive positioning of different targets in a wide range, which plays important role for the fast and precise positioning of the UAV.

\section{Position circuit summarization}

Launching the aerial photos or videos in the UAV shooting target area which is to be located, at the same time, obtaining POS data of the UAV at the moment. 
Firstly, using optical flow algorithm Gaussian pyramid method to stitch real-time UAV aerial photos or video frames and extract the overlapping parts.

Secondly, making the use of POS data to aided aerial triangulation measurement to determine the ground objects three-dimensional coordinates of the overlapping part, and then calculate the ground objects two-dimensional coordinates of the entire aerial photos or video frames. According to the searching tactics developed in this article, finding the matching area on the satellite digital map.

Finally, taking advantage of the Gaussian pyramid optical flow algorithm, the aerials or video frames are matched with the digital satellite map in this range, while getting the location information from digital satellite map to complete the targeting.

The method uses the advantage of POS and satellite digital map, which is that getting location information quickly, achieving targeting real-time and exactly in a wide range, while avoiding the introduction of ground control points, the error influence of the orthographic correction and POS systems, which improves the targeting accuracy and speed, which is an effective targeting method using image matching based POS.

\section{Gaussian pyramid matching method based on optical flow}

The degree of overlap of UAV video frame is large, the amount of motion between frames is small, so making use of optical flow method can register quickly and accurately. The Optical flow method approximately calculates motion field which can't be obtained directly in image sequence through optical flow field, then estimating the relative motion between images according to motion field. This method is based on parametric modeling, which can describe the actual motion process better and avoid the problem of inaccurate motion vectors that is what hierarchical block matching method exists.

Assuming that the motion field of the image $f(x, t)$ with respect to the image $f\left(x_{t}, t\right)$ is $p=\left(p_{x}\right.$, $\left.\mathrm{p}_{\mathrm{y}}\right)=\mathrm{x}-\mathrm{x}_{\mathrm{t}}$. And $\mathrm{p}_{\mathrm{x}}, \mathrm{p}_{\mathrm{y}}$ respectively represents the motion amount of horizontal and vertical directions. Motion field estimation makes the following sum of squared error Err reach minimum value:

$$
\operatorname{Err}=\sum\left(\mathrm{f}(\mathrm{x}, \mathrm{t})-\mathrm{f}\left(\mathrm{x}-\mathrm{p}, \mathrm{t}_{\tau}\right)\right)^{2}
$$

If the motion model is affine motion model:

$$
\begin{aligned}
& \sum\left[\frac{\partial \mathrm{f}(\mathrm{x}, \mathrm{t})}{\partial \mathrm{x}}\right]^{2} \\
& \sum \mathrm{x}\left[\frac{\partial \mathrm{f}(\mathrm{x}, \mathrm{t})}{\partial \mathrm{x}}\right]^{2} \\
& \sum \mathrm{y}\left[\frac{\partial \mathrm{f}(\mathrm{x}, \mathrm{t})}{\partial \mathrm{x}}\right]^{2} \\
& \sum x\left[\frac{\partial f(x, t)}{\partial x}\right]^{2} \\
& \sum \mathrm{x}^{2}\left[\frac{\partial \mathrm{f}(\mathrm{x}, \mathrm{t})}{\partial \mathrm{x}}\right]^{2} \\
& \sum \mathrm{y}\left[\frac{\partial \mathrm{f}(\mathrm{x}, \mathrm{t})}{\partial \mathrm{x}}\right]^{2} \\
& \sum x y\left[\frac{\partial f(x, t)}{\partial x}\right]^{2} \\
& \sum x y\left[\frac{\partial f(x, t)}{\partial x}\right]^{2} \\
& \sum \mathrm{y}^{2}\left[\frac{\partial \mathrm{f}(\mathrm{x}, \mathrm{t})}{\partial \mathrm{x}}\right]^{2} \\
& \sum \frac{\partial \mathrm{f}(\mathrm{x}, \mathrm{t})}{\partial \mathrm{x}} \frac{\partial \mathrm{f}(\mathrm{x}, \mathrm{t})}{\partial \mathrm{y}} \\
& \sum \mathrm{x} \frac{\partial \mathrm{f}(\mathrm{x}, \mathrm{t})}{\partial \mathrm{x}} \frac{\partial \mathrm{f}(\mathrm{x}, \mathrm{t})}{\partial \mathrm{y}} \\
& \sum \mathrm{x} \frac{\partial \mathrm{f}(\mathrm{x}, \mathrm{t})}{\partial \mathrm{x}} \frac{\partial \mathrm{f}(\mathrm{x}, \mathrm{t})}{\partial \mathrm{y}} \\
& \sum x^{2} \frac{\partial f(x, t)}{\partial x} \frac{\partial f(x, t)}{\partial y} \\
& \left.\sum \mathrm{y} \frac{\partial \mathrm{f}(\mathrm{x}, \mathrm{t})}{\partial \mathrm{x}} \frac{\partial \mathrm{f}(\mathrm{x}, \mathrm{t})}{\partial \mathrm{y}}\right] \\
& \sum \frac{\partial \mathrm{f}(\mathrm{x}, \mathrm{t})}{\partial \mathrm{x}} \frac{\partial \mathrm{f}(\mathrm{x}, \mathrm{t})}{\partial \mathrm{y}} \quad \sum \mathrm{x} \frac{\partial \mathrm{f}(\mathrm{x}, \mathrm{t})}{\partial \mathrm{x}} \frac{\partial \mathrm{f}(\mathrm{x}, \mathrm{t})}{\partial \mathrm{y}} \quad \sum \mathrm{y} \frac{\partial \mathrm{f}(\mathrm{x}, \mathrm{t})}{\partial \mathrm{x}} \frac{\partial \mathrm{f}(\mathrm{x}, \mathrm{t})}{\partial \mathrm{y}} \\
& \sum \mathrm{x} \frac{\partial \mathrm{f}(\mathrm{x}, \mathrm{t})}{\partial \mathrm{x}} \frac{\partial \mathrm{f}(\mathrm{x}, \mathrm{t})}{\partial \mathrm{y}} \sum \mathrm{x}^{2} \frac{\partial \mathrm{f}(\mathrm{x}, \mathrm{t})}{\partial \mathrm{x}} \frac{\partial \mathrm{f}(\mathrm{x}, \mathrm{t})}{\partial \mathrm{y}} \sum \mathrm{xy} \frac{\partial \mathrm{f}(\mathrm{x}, \mathrm{t})}{\partial \mathrm{x}} \frac{\partial \mathrm{f}(\mathrm{x}, \mathrm{t})}{\partial \mathrm{y}} \\
& \sum \mathrm{y} \frac{\partial \mathrm{f}(\mathrm{x}, \mathrm{t})}{\partial \mathrm{x}} \frac{\partial \mathrm{f}(\mathrm{x}, \mathrm{t})}{\partial \mathrm{y}} \\
& \sum x y \frac{\partial f(x, t)}{\partial x} \frac{\partial f(x, t)}{\partial y} \\
& \sum \mathrm{y}^{2} \frac{\partial \mathrm{f}(\mathrm{x}, \mathrm{t})}{\partial \mathrm{x}} \frac{\partial \mathrm{f}(\mathrm{x}, \mathrm{t})}{\partial \mathrm{y}} \\
& \sum \mathrm{y} \frac{\partial \mathrm{f}(\mathrm{x}, \mathrm{t})}{\partial \mathrm{x}} \frac{\partial \mathrm{f}(\mathrm{x}, \mathrm{t})}{\partial \mathrm{y}} \\
& \sum x y \frac{\partial f(x, t)}{\partial x} \frac{\partial f(x, t)}{\partial y} \\
& \sum\left[\frac{\partial \mathrm{f}(\mathrm{x}, \mathrm{t})}{\partial \mathrm{y}}\right]^{2} \\
& \sum \mathrm{x}\left[\frac{\partial \mathrm{f}(\mathrm{x}, \mathrm{t})}{\partial \mathrm{y}}\right]^{2} \\
& \sum \mathrm{y}\left[\frac{\partial \mathrm{f}(\mathrm{x}, \mathrm{t})}{\partial \mathrm{y}}\right]^{2} \\
& \sum \mathrm{x}\left[\frac{\partial \mathrm{f}(\mathrm{x}, \mathrm{t})}{\partial \mathrm{y}}\right]^{2} \\
& \sum \mathrm{x}^{2}\left[\frac{\partial \mathrm{f}(\mathrm{x}, \mathrm{t})}{\partial \mathrm{y}}\right]^{2} \\
& \sum x y\left[\frac{\partial f(x, t)}{\partial y}\right]^{2} \\
& \begin{array}{l}
\sum x y \frac{\partial f(x, t)}{\partial x} \frac{\partial f(x, t)}{\partial y} \\
\sum y^{2} \frac{\partial f(x, t)}{\partial x} \frac{\partial f(x, t)}{\partial y} \\
\sum y\left[\frac{\partial f(x, t)}{\partial y}\right]^{2} \\
\sum x y\left[\frac{\partial f(x, t)}{\partial y}\right]^{2} \\
\sum y^{2}\left[\frac{\partial f(x, t)}{\partial y}\right]^{2}
\end{array} \mid\left[\begin{array}{l}
a_{x} \\
b_{x} \\
c_{x} \\
b_{y} \\
c_{y}
\end{array}\right]=- \\
& =-\left[\begin{array}{c}
\sum \frac{\partial \mathrm{f}(\mathrm{x}, \mathrm{t})}{\partial \mathrm{x}} \frac{\partial \mathrm{f}(\mathrm{x}, \mathrm{t})}{\partial \mathrm{t}} \\
\sum \mathrm{x} \frac{\partial \mathrm{f}(\mathrm{x}, \mathrm{t})}{\partial \mathrm{x}} \frac{\partial \mathrm{f}(\mathrm{x}, \mathrm{t})}{\partial \mathrm{t}} \\
\sum \mathrm{y} \frac{\partial \mathrm{f}(\mathrm{x}, \mathrm{t})}{\partial \mathrm{x}} \frac{\partial \mathrm{f}(\mathrm{x}, \mathrm{t})}{\partial \mathrm{t}} \\
\sum \frac{\partial \mathrm{f}(\mathrm{x}, \mathrm{t})}{\partial \mathrm{y}} \frac{\partial \mathrm{f}(\mathrm{x}, \mathrm{t})}{\partial \mathrm{t}} \\
\sum \mathrm{x} \frac{\partial \mathrm{f}(\mathrm{x}, \mathrm{t})}{\partial \mathrm{y}} \frac{\partial \mathrm{f}(\mathrm{x}, \mathrm{t})}{\partial \mathrm{t}} \\
\sum \mathrm{y} \frac{\partial \mathrm{f}(\mathrm{x}, \mathrm{t})}{\partial \mathrm{y}} \frac{\partial \mathrm{f}(\mathrm{x}, \mathrm{t})}{\partial \mathrm{t}}
\end{array}\right]
\end{aligned}
$$

Making the derivative of the above formula zero, the motion estimation of image $f(x, t)$ can be obtained.

After the initial motion estimation, the image displacement compensates motion volume estimated. The matching process conduct repeatedly between other frame and the target frame to obtain the residual amount of motion estimation. Iterating is not stopped until the residual motion volume is below the initial threshold value. In the course of each iteration, the function frame will be multiplied by a fixed size Gaussian function, and is sampled down in a fixed ratios of length and width to increase the speed. After many experiments, the author, ensure when the ratio is 0.5 , the speed is fastest. Other frames will get transferred to the target frame after getting the transformation matrix. Make sure the relative position between the images according to the overlapping area, and then stitching images. 


\section{An auxiliary aerial triangulation using the POS}

UAV's Position and Orientation System (namely POS) can obtain the images, while it can directly determine the three-dimensional coordinates of the photo site, as well as the attitude azimuth of the aircraft at the moment of shooting aerials ${ }^{[3]}$. POS auxiliary aerial triangulation method is also called Integrated Sensor Orientation (ISO), which is through the observational data of the POS system is united and processed to directly determine the position and attitude of the aerial instrument (Dynamic Kalman Filtering), which is done simultaneous adjustment with observation of the image point coordinates, so as to integrally ensure the three-dimensional spatial coordinates and six exterior orientation elements of ground target points to realize photogrammetric block adjustment of little or no ground control points ${ }^{[4]}$.

In order to study the corresponding mathematical relationship of the image point and the ground point, firstly, the conformation equation of the central projection is established. Setting up the image point $\mathrm{a}$ is $(\mathrm{x}, \mathrm{y})$ in image plane coordinate $\mathrm{o}-\mathrm{xyz}$ which considers $\left(\mathrm{x}_{0}, \mathrm{y}_{0}\right)$ as the main point of the photo, the ground coordinate of the object point $\mathrm{A}$ is $(\mathrm{X}, \mathrm{Y}, \mathrm{Z})$ in object spatial coordinates $\mathrm{M}-\mathrm{XYZ}$, the ground coordinate of the center of projection $S$ is $\left(X_{S}, Y_{S}, Z_{S}\right)$ in object spatial coordinates $\mathrm{M}-\mathrm{XYZ}$, then the object spatial coordinate and the image plane coordinate need satisfy theoretically the collinear condition equation ${ }^{[5]}$ that the center of projection, the image point and the object point is in the same straight line:

$$
\left\{\begin{array}{l}
x-x_{0}=-f \frac{a_{1}\left(X-X_{S}\right)+b_{1}\left(Y-Y_{S}\right)+c_{1}\left(Z-Z_{S}\right)}{a_{3}\left(X-X_{S}\right)+b_{3}\left(Y-Y_{S}\right)+c_{3}\left(Z-Z_{S}\right)} \\
y-y_{0}=-f \frac{a_{2}\left(X-X_{S}\right)+b_{2}\left(Y-Y_{S}\right)+c_{2}\left(Z-Z_{S}\right)}{a_{3}\left(X-X_{S}\right)+b_{3}\left(Y-Y_{S}\right)+c_{3}\left(Z-Z_{S}\right)}
\end{array}\right.
$$

In formula (3), $a_{i}, b_{i}, c_{i}(i=1,2,3)$ is called the direction cosine, which can be calculated through the diagonal elements of POS.

Because a image point of the photo can only compose two equations, the unknown numbers are three, we must photograph for the same object from different photo stations to obtain images with a certain degree of overlap. Based on the conformational equation, the image points with the same name of each images separately compose the conditions equation, the formula (4) can be obtained from the dimensional relative formula.

$$
\left\{\begin{array}{l}
\frac{X-X_{s}}{Z-Z_{S}}=\frac{a_{1} x_{0}+a_{2} y_{0}-a_{3} f}{c_{1} x_{0}+c_{2} y_{0}-c_{3} f} \\
\frac{Y-Y_{S}}{Z-Z_{S}}=\frac{b_{1} x_{0}+b_{2} y_{0}-b_{3} f}{c_{1} x_{0}+c_{2} y_{0}-c_{3} f} \\
\frac{X-X_{s}^{\prime}}{Z-Z_{S}^{\prime}}=\frac{a_{1}^{\prime} x_{1}+a_{2}^{\prime} y_{1}-a_{3}^{\prime} f}{c_{1}^{\prime} x_{1}+c_{2}^{\prime} y_{1}-c_{3}^{\prime} f} \\
\frac{Y-Y_{s}^{\prime}}{Z-Z_{S}^{\prime}}=\frac{b_{1}^{\prime} x_{1}+b_{2}^{\prime} y_{1}-b_{3}^{\prime} f}{c_{1}^{\prime} x_{1}+c_{2} y_{1}-c_{3}^{\prime} f}
\end{array}\right.
$$

Solving the equation, three-dimensional coordinates of the corresponding object point $(\mathrm{X}, \mathrm{Y}, \mathrm{Z})$ can be obtained. Using block adjustment error equation of POS auxiliary beam method, the orientation parameters and coordinate observation of the image points of the images are done simultaneous adjustment to improve the accuracy.

\section{Determine the matching area}

To reduce the matching time, making the matching area small enough, but the selected area should fully include aerials or video frames, then developing the following tactics. The flight altitude of the UAV is generally a few hundred meters to several kilometers, for the flat ground, the earth objects in aerial photos or video frames feature can be approximated to the same plane, the coordinates can be obtained based on the overlapping area. Establishing Cartesian coordinate 
system in the picture plane to calculate the unknown coordinates of the non-overlapping regions so as to get four corner points of the aerial, respectively, $\left(\mathrm{x}_{1}, \mathrm{y}_{1}\right)\left(\mathrm{x}_{2}, \mathrm{y}_{2}\right)\left(\mathrm{x}_{3}, \mathrm{y}_{3}\right)\left(\mathrm{x}_{4}, \mathrm{y}_{4}\right)$,. Setting up that the distance of this type of UAV use ISO positioning error is $S$, as shown in the picture, on the satellite digital map, considering the four corner points as the center, thinking of $S$ as the radius to draw circle and connect the round external tangents, then intersecting at four points, obtaining the four points coordinates $(\mathrm{x}, \mathrm{y})$, the formula is as follows:

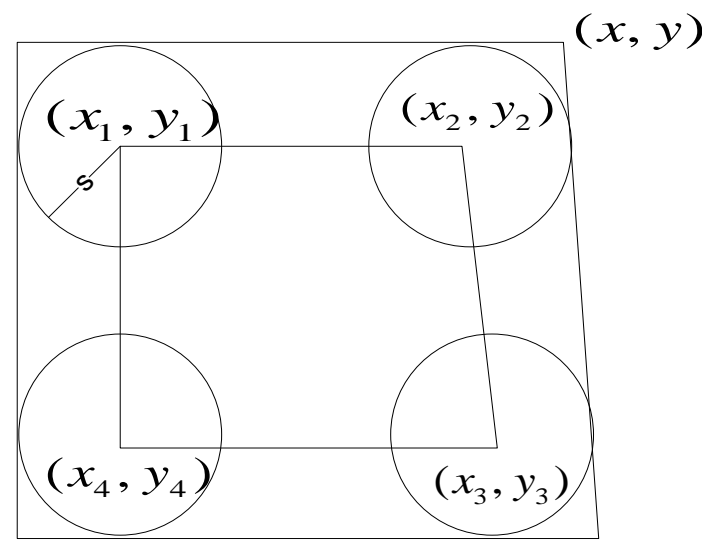

Fig.2 The diagram of mapping range

$$
\left\{\begin{array}{l}
x=x_{i} \pm s \frac{\sqrt{\left(\frac{y_{k}-y_{i}}{x_{k}-x_{i}}\right)^{2}+1}-\sqrt{\left(\frac{y_{j}-y_{i}}{x_{j}-x_{i}}\right)^{2}+1}}{\frac{y_{k}-y_{i}}{x_{k}-x_{i}}-\frac{y_{j}-y_{i}}{x_{j}-x_{i}}} \\
y=y_{i} \pm s \frac{\sqrt{\left(\frac{x_{k}-x_{i}}{y_{k}-y_{i}}\right)^{2}+1}-\sqrt{\left(\frac{x_{j}-x_{i}}{y_{j}-y_{i}}\right)^{2}+1}}{\frac{x_{k}-x_{i}}{y_{k}-y_{i}}-\frac{x_{j}-x_{i}}{y_{j}-y_{i}}}
\end{array}\right.
$$

The j, i, k respectively1, 2, 3. 2, 3, 4. 3, 4, 1. 4, 1, 2. The solutions of each (x, y) calculated have two, choosing the four values which form quadrilateral. Marking the four points and lining to form a closed figure, the area is the search area.

\section{The simulation analysis}

As is shown in Figure 6, The test images are two aerial of one type of one UAV in Tang County, Shijiazhuang, Hebei Province $(2000 \times 3000)$ and google digital map. The flight with overlapping rate of the aerial is low, which can illustrate the feasibility of method proposed in this paper better. Taking advantage of the Gaussian pyramid optical flow algorithm, two aerial photos are spliced, as is shown in fig a. According to ISO to determine the three-dimensional coordinates, obtaining the exterior orientation elements through reading the * .txt file. And calculating the coordinates of the overlap region ground point (X, Y, Z), finding the four corner points on a digital aerial satellite map after adjustment. The ISO positioning error distance is transformed according to the scale to be considered as the error radius. Then calculating the coordinates of the four corners of the matching region by the formula (3), extracting the area, the results are shown in Figure b. Using the Gaussian pyramid aerial optical flow algorithm, the aerial is registered which think of the region as the target so as to gain precise location information of the whole aerial. The results are shown in c, taking2.73 seconds. 

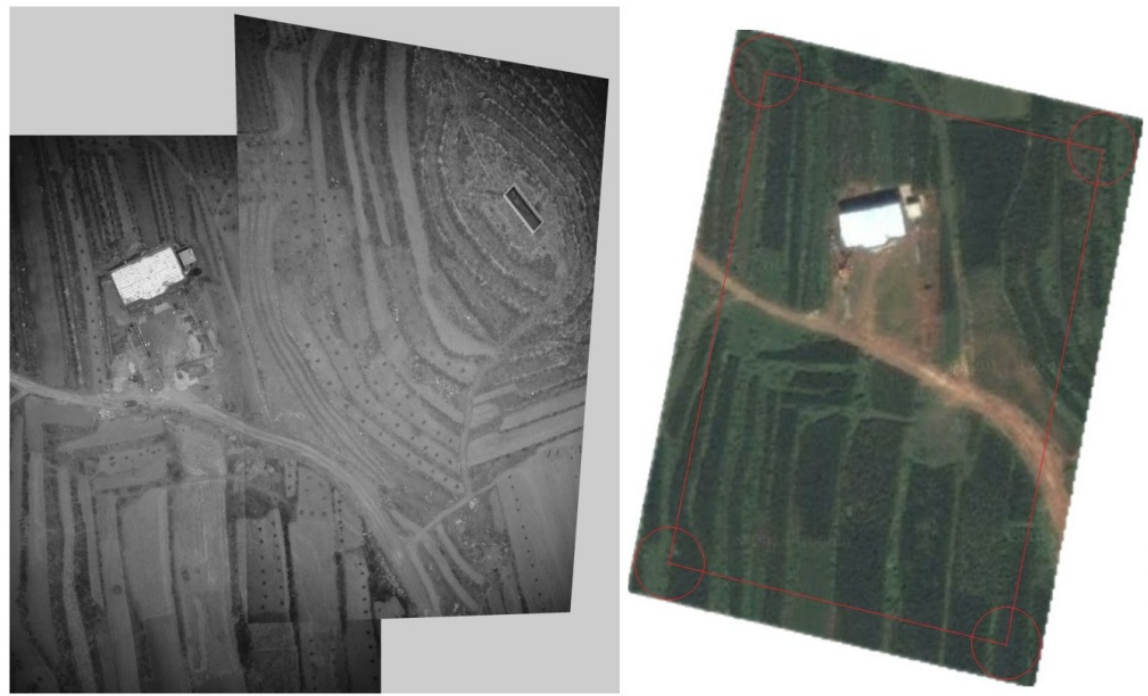

(a)The registration results of aerial photographs and digital maps (c)Reading coordinate information Fig.6 the results of region confirming

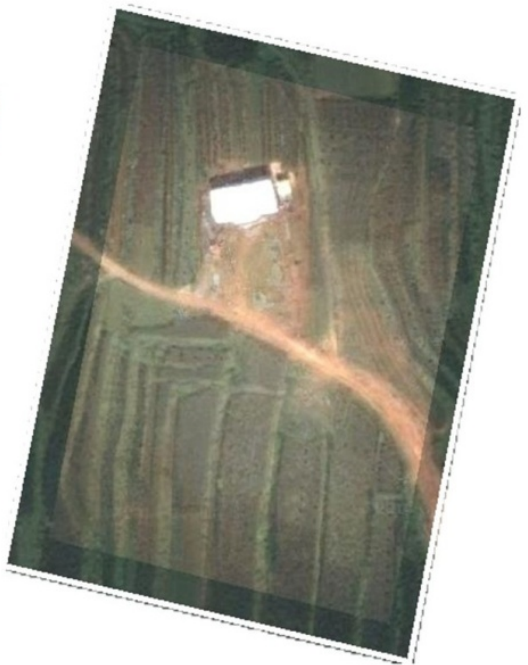

(b) Registration details

\section{Conclusion}

Based on the POS, this paper presents a method that is UAV image matching targeting, using of the ISO and the Gaussian pyramid optical flow algorithm, which develops a search strategy that mainly aim to the two-dimensional target positioning. Avoiding leading into the ground control points and ortho Correction, this paper improves accuracy and speed of the UAV target location.

Firstly, the combining features of the ISO and digital satellite map, which expands the scope of application of the base map.

Secondly, developing the search strategies of the aerial photo or video frame in the digital satellite map, which reduces the matching range and improves the positioning speed.

The algorithm proposed in this paper has shortcomings: when calculating the unknown coordinates of the non-overlapping regions using ISO to coarse locate, the altitude of UAV needs very high, the ground needs relatively flat and the complex situation can't be handled. When the applying the Gaussian pyramid optical flow algorithm, the matching error of urban high-rise buildings exists. These problems will be improved in the following study.

\section{References}

[1] Mao Zhaojun, Wang Dehu. A model of target position for UAV based on attitude measuring /laser range-finder[J]. Fire Control \& Command Control. 2003, 28(5): 334-3379.

[2] Srikanth Saripalli, James F. Montgomery. Vision based autonomous landing of an unmanned aerial vehicle[C]. Proceeding Of IEEE International Conference On Robotics And Automation. May, 2005.

[3] Lu Heng, Li Yongshu. A Method of Automatic Extraction of Image Control Points for UAV Image Based on POS Data[J]. Opto-Electronic Engineering, 2011, 38(9): 25-29.

[4] Yuan Xiuxiao. POS supported Bundle Block Adjustment[J]. Acta Geodaetica et Cartographica Sinica, 2008, 37(3): 343-348.

[5] WANG Zhizhuo. Principle of Photogrammetry(with Remote Sensing)[M]. Wuhan: Press of Wuhan Technical University of Surveying and Mapping, Beijing: Publishing House of Surveying and Mapping, 1990. 